\title{
A Rayleigh Backscattering Noise Resilient and Cost Effective Single Fiber WDM-PON Scheme using DQPSK and Intensity Re-modulation Technique
}

\author{
Muhammad Arshad \\ UET Peshawar
}

\author{
Nasir Saleem, PhD \\ ICT, Islamabad
}

\author{
Shahzada Alamgir Khan, \\ $\mathrm{PhD}$ \\ ICT, Islamabad
}

\begin{abstract}
In this research work, we propose and simulate a Rayleigh backscattering noise-resilient and cost-effective scheme of standard single mode SSM, single fiber bi-directional optical access network using wavelength division multiplexed passive optical network (WDM-PON) technology. A differential quadrature phase shift keying (DQPSK) optical signal is used at optical line terminal (OLT) for downstream (DS) communication and intensity re-modulation technique is used at optical network unit (ONU) in upstream (US) optical signal, while using centralized laser source and no additional laser at terminals. Simulation setup is prepared in Opt-system 13 and results show that on the aggregate $100 \mathrm{Gbps}$ downstream transmission and 25 Gbps upstream communications for 10 ONUs can be successfully achieved over a longer bidirectional standard single mode fiber (SSMF). It is also observed that proposed single fiber based bidirectional WDM-PON has lower transmission power losses while ensuring high resilience against Rayleigh backscattering (RB) noise and improved receiver sensitivity in both directions of transmission. (Abstract)
\end{abstract}

\section{Keywords}

Centralized Light Source; Wavelength Division Multiplexing Passive Optical Network (WDM-PON); Rayleigh Backscattering; Differential Quadrature Phase Shift Keying (DQPSK) Inverse Return-To-Zero (IRZ); Receiver Sensitivity; Optical; communications; Noise mitigation insert (key words)

\section{INTRODUCTION}

Due to the rapid growth of triple play service and bandwidthhungry applications on the Internet, various structures of optical access network have been proposed to realize fiber to the home (FTTH) service. Among various optical access networks, wavelength division multiplexing passive optical network (WDM-PON) is regarded as one of the most promising scheme to accord with the rapid growth of bandwidth requirement [1]. As per Cisco forecast project, during 2015 and 2020 global internet traffic will grow at a compound annual growth rate (CAGR) of 22 percent from 2015 to 2020 . Video traffic will be 82 percent of all consumer Internet traffic by 2020, up from 70 percent in 2015 [2]. Currently, high quality internet services like video calling, video conferencing, high definition television (HDTV), 3-D videos, and other services have become quite prevalent. In this regard, Wavelength division multiplexing passive optical networks (WDM-PONs) are recognized as promising candidate in next generation access (NGA) networks, to meet the current and future bandwidth demands of explosive growth of internet traffic in access network. Moreover in WDM-PON, high capacity services can be accessed by every subscribers, each optical network unit (ONU) has dedicated bandwidth which can be readily scalable as per requirement, traffic restoration, system and network capacity can be upgraded, centralized laser source and re-modulation features can be achieved to deploy a cost-effective access network. [3]. Deployment cost of the WDM-PON can further be reduced by using a single fiber for both downstream and upstream in bidirectional transmission [4]. When a single fiber is used for bidirectional transmission in loop-back structure, Rayleigh backscattering (RB) noise is inevitable and induces serious interference in upstream direction [5]. Rayleigh backscattering (RB) noise and induced due to the intrinsic nature of the fiber with microscopic fluctuations of refractive index This drawback directly limits both the transmission distance and receiver sensitivity. There are two kinds of RB noise in single fiber loop-back structure [6]. They are the carrier RB noise and the signal RB noise. Carrier RB noise is generated from beating between the upstream signal and the reflected downstream signal of the same wavelengthThere are two sources of RB noise generation in standard single mode fiber (SSMF) bidirectional WDM-PON, first one is backscattered light of downstream optical source and other one is backscattered light of up- stream modulated signal. The first source of RB noise can influence both downstream as well as upstream and the second source of RB noise can influence upstream more severely. In reflective silicon optical amplifier (RSOA) based single fiber bidirectional WDMPON, the upstream trans- mission is more affected because backscattered light is re-amplified at ONU by RSOA. Hence, in single fiber centralized laser source WDM-PON, RB noise causes more degradation in upstream transmission due to influence of both RB noise sources [7].

Recently, several techniques have been reported in centralized laser-source WDM-PON for downstream and upstream transmission on single-fiber to suppress the R.B. noise induced effects. R.S.O.A. based WDM-PON techniques has been proposed for enhance performance by minimizing R.B. noise such as, applying chirping-clipping effects [8], using multi-wavelength source in a service-ONU [9], a novel DPSK downstream and re-modulated OOK upstream [10], pulse broadening by chirped R.Z. modulation [11], cross-seeding scheme in WDM based NGA Q.Guo, noise predictive equalizer with noise canceller for RSOA based WDM-PON [12] and signal re-modulation ring with RB interferometric noise mitigation in WDM-PON [13]. Further techniques based on carrier-distributed WDM-PONs using in-band optical filtering[13], line coding with electrical filtering in PON networks [14], high extinction ratio (ER) IRZ downstream and phase re-modulated DPSK upstream [15] and DPSK downstream and OOK intensity re-modulated up- stream [16] have also been proposed to reduce the influence of $\mathrm{RB}$ induced noise in WDM-PON. Although, enhanced resilience 
against RB induced noise has been observed by these techniques but they reduce effectiveness due to one or more of these reasons, either design complexity, additional components requirements, high deployment cost, limitations of data rate or low receiver sensitivity in downstream and upstream. Furthermore, employing dispersion compensated fiber (DCF) and erbium doped fiber amplifier (EDFA) are not preferred for the cost effective architecture in $20-25 \mathrm{~km}$ reach PON.

In this paper, we propose and demonstrate a cost-effective and Rayleigh-backscattering noise-resilient design of single-fiber centralized light-source bidirectional WDM-PON with improved receiver-sensitivity. A $10 \mathrm{Gbit} / \mathrm{s}$ DQPSK data signal is used at O.L.T for downstream without pulse carving, whereas a $2.5 \mathrm{Gbit} / \mathrm{s}$ IRZ data signal is used for upstream signal by intensity-re-modulation of downstream signal, no additional laser is used at ONU. Resilience against R.B. induced noise for I.R.Z. upstream signal can be achieved by reducing modulation-index of downstream DQPSK signal. Simulation results show that aggregated $100 \mathrm{Gbit} / \mathrm{s}$ downstream transmissions of 10 DQPSK channels and aggregated 25Gbit/s upstream-transmission of 10 I.R.Z. channels, using $100 \mathrm{GHz}$ channel spacing, can be successfully achieved in $25 \mathrm{~km}$ bidirectional S.S.M.F. Further it is observed that proposed single-fiber based bidirectional WDM-PON has lower transmission power-penalties which ensure high resilience against R.B. induced noise and also improved receiver-sensitivity is obtained in both directions of transmission. As compared to previous works, DQPSK is advanced modulation technique for multi-level, spectrallyefficient and high data-rate transmission. Since it transmits 2 bits per symbol therefore only half spectral occupancy is required than DPSK. The rest of the paper comprises these sections. Section 2 explains working principle and architecture, section 3 describes simulation setup, section 4 covers transmission performance analysis and finally conclusions.

\section{PRINCIPLE AND SYSTEM SETUP}

Principle of the proposed carrier RB noise mitigation scheme is illustrated in Fig. 1. When continuous wave (CW) seeding light is used in downstream direction, upstream signal and the reflected downstream signal will interfere and beat in the low frequency region, as illustrated in the RF spectrum in Fig. 1. A thicker red arrow is used to indicate upstream signal modulation, while a thin black arrow is used to represent the carrier RB noise. In order to suppress this low frequency interference, microwave frequency $\mathrm{f} \mathrm{GHz}$ is modulated onto the downstream wavelength to generate a multi-subcarrier downstream signal. Owing to the gain saturation effect of cascaded-SOA, central carrier of the upstream signal is suppressed and this avoids interfering with the reflected downstream central wavelength at low frequency region. However, interference of the RB noise with other subcarriers is increased and this interference appears at frequencies that are the integer multiples of $\mathrm{f} \mathrm{GHz}$ (e.g. $\mathrm{f} \mathrm{GHz}, 2 \mathrm{f} \mathrm{GHz}$ ). Thus, a two-tap MPF with periodical notches at $\mathrm{f} \mathrm{GHz}$ can be used to suppress those enhanced high frequency interference as well as the residual $\mathrm{f} \mathrm{GHz}$ microwave signal in the upstream direction.

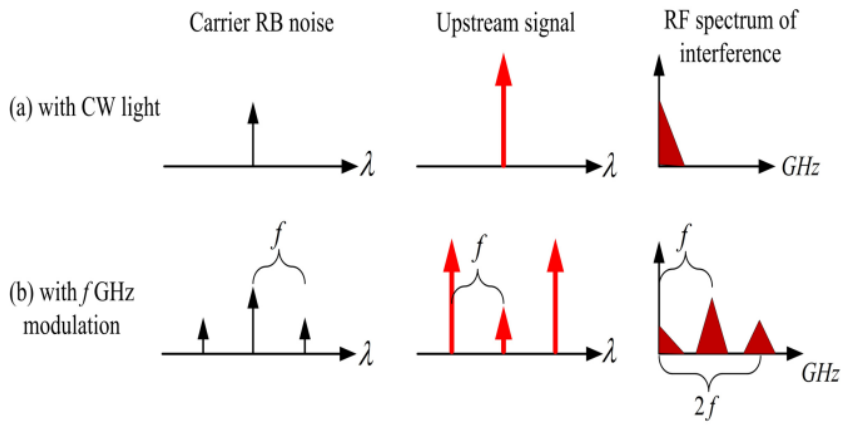

Fig. 1. Theory of carrier RB noise mitigation techniques.

The proposed loop-back WDM-PON system is shown in Fig. 2. In single fiber bidirectional transmission system, Rayleigh backscattering noise can be reduced by using different wavelengths in upstream and downstream directions. However, this approach increases both the cost and complexity for operation and maintenance. In our system, the downstream seeding lights are generated by the $\mathrm{CW}$ laser sources named LD 1 to LD $\mathrm{n}$. All the downstream seeding lights are multiplexed at the arrayed waveguide grating (AWG) and are then modulated by an MZM with a fixed frequency at $\mathrm{f} \mathrm{GHz}$. A modulated signal with multi-subcarrier is generated. After transmitting through a $45-\mathrm{km} \mathrm{SMF}$, the downstream seeding lights are de-multiplexed at the remote node (RN) by an AWG and are sent to the corresponding ONU. A 0.8 km SMF is connected between RN and ONU.

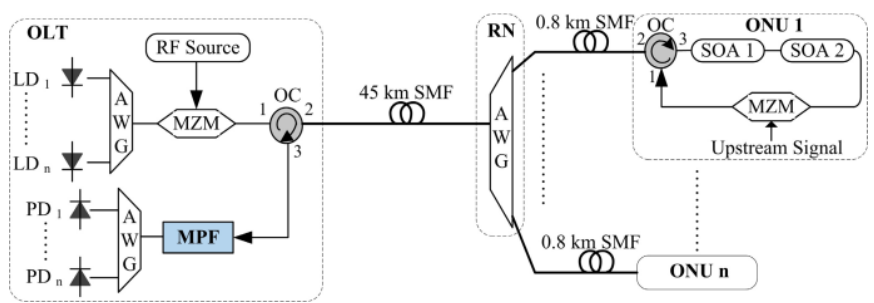

Fig. 2. Proposed setup of the 10-Gb/s loop-back WDMPON. LD: laser diode, AWG: arrayed waveguide grating, OC: optical circulator, MZM: Mach-Zehnder modulator, RF source: radio frequency source, SMF: single mode fiber, SOA: semiconductor optical amplifier, MPF: microwave photonic filter, PD: photodiode.

In the ONU, the seeding light is first passed through the cascaded-SOA for central carrier suppression, and then the upstream data is modulated onto the carrier-suppressed signal by an MZM. With the help of photonic integrated circuit (PIC) technology, 10Gb/s low cost MZM can be monolithically integrated with SOA easily. This monolithically photonic integrated circuit improves the system cost, size and power consumption to bring better support for realizing cost-effective optical access network [17, 18]. The upstream signal is then transmitted back to the OLT via an optical circulator for receiving. In order to suppress the residual $\mathrm{f} \mathrm{GHz}$ microwave signal and the increase $\mathrm{RB}$ interference at $\mathrm{f} \mathrm{GHz}$, the upstream signal is first launched to a microwave photonic filter that has a deep notch at $\mathrm{f} \mathrm{GHz}$ before detecting with a photodiode (PD). Due to the wavelength independent characteristic of MPF, all upstream channels can share this MPF to remove residual interference noise without increasing system cost. Unlike MZM based carrier suppression scheme that is sensitive to bias drifting, our carrier suppression scheme utilizes gain saturation effect in SOA and is more stable over time and less sensitive to environmental temperature change. Furthermore, polarization 
independent central carrier suppression approach can be realized if polarization independent SOA is used in our system.

\section{WORKING PRINCIPLE AND PROPOSED ARCHITECTURE}

Before when cascaded-SOA is used to realize central carrier suppression, the resultant central carrier suppression ratio (CCSR) depends on the gain saturation effect of SOA. Compared to single stage SOA, the overall gain saturation effect is strengthened by the cascaded-SOA structure [19]. It has been shown that a better high-pass filtering effect can be achieved by the strengthened gain saturation effect in cascaded-SOA [20]. In our scheme, we make use of this phenomenon such that a high suppression ratio for baseband frequency of the microwave modulated signal is achieved. Therefore, central carrier of the multi-subcarrier optical signal is suppressed effectively after passing the cascaded-SOA. To evaluate the high-pass filtering effect, we measure the opticalto-optical frequency response curve of cascaded-SOA under different input optical power and use single stage SOA for comparison, as shown in Fig. 3 (a). The bias currents for SOA 1 and SOA 2 in cascaded-SOA structure are $280 \mathrm{~mA}$ and 380 $\mathrm{mA}$, respectively. In single stage SOA, the bias current is fixed at $380 \mathrm{~mA}$. As shown in Fig. 3 (a), the low frequency suppression ratio is enhanced significantly as the input optical power increases from $-12 \mathrm{dBm}$ to $-4 \mathrm{dBm}$. When the input optical power is at $-4 \mathrm{dBm}$, baseband suppression ratio is over $45 \mathrm{~dB}$, which provides a high suppression ratio for the central carrier. Compared to single stage SOA, baseband suppression ratio is improved by $\geq 25 \mathrm{~dB}$ under the same input power $(-4 \mathrm{dBm})$. Since the total gain saturation effect is enhanced by the cascaded-SOA, carrier lifetime of SOAs are reduced which corresponding to a deeper filtering curve in low frequency region. In our experiment, the downstream signal power is fixed around $1 \mathrm{dBm}$ and the input optical power to the cascaded-SOA is about $-8 \mathrm{dBm}$ after transmitting through the $45 \mathrm{~km} \mathrm{SMF}$. According to Fig. 3 (a), a baseband suppression ratio of $25 \mathrm{~dB}$ can be achieved.

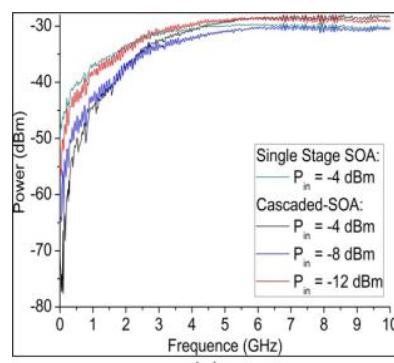

(a)

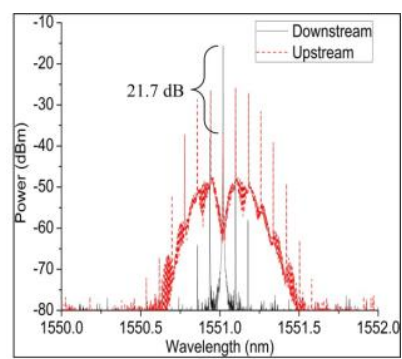

(b)
Fig. 3. (a) Saturation graphs of one stage SOA and cascade-SOA, (b) Related optical spectra.

The comparison of optical spectra between downstream and upstream signal is shown in Fig. 3 (b). Modulation frequency of the radio frequency (RF) source in OLT is fixed at $10 \mathrm{GHz}$, and the upstream data is a $10 \mathrm{~Gb} / \mathrm{s} 231-1$ pseudo random binary sequence (PRBS) signal. Compared with the original downstream multi-subcarrier signal, central carrier of the upstream signal is suppressed by $21.7 \mathrm{~dB}$ through the use of cascaded-SOA in ONU. Figure 4 (a) and (b) are used for measuring OSRNR and BER performances, respectively. In Fig. 4 (a), CW light at $1551.02 \mathrm{~nm}$ and optical power of 13 $\mathrm{dBm}$ is used as the downstream light. A $10 \mathrm{GHz}$ microwave signal is modulated onto this CW light through an MZM as a multi-subcarrier downstream seeding light. The downstream signal is split and combined using two optical couplers to mimic beating interference between the carrier RB noise and the upstream signal. In the lower branch, carrier RB noise is generated by launching the signal into a $45-\mathrm{km}$ SMF through an optical circulator. The carrier RB noise is amplified by erbium doped fiber amplifier (EDFA), while the corresponding amplified spontaneous emission (ASE) noise is filtered by an optical band pass filter. Since beating is a polarization sensitive phenomenon, a polarization controller is used to maximize the beating between the downstream and upstream signal. In the upper branch, we use cascaded-SOA structure to generate the central carrier suppressed signal. SOA 1 is a linear optical amplifier (LOA) and SOA 2 is a nonlinear SOA (NL-SOA). The LOA is mainly for linear amplification of the input signal and NL-SOA is mainly for gain saturation effect. A $10 \mathrm{~Gb} / \mathrm{s}$ 231-1 PRBS upstream signal is modulated onto the carrier suppressed signal using an MZM. Optical spectrum of the generated upstream signal is shown by the red curve in Fig. 3 (b).

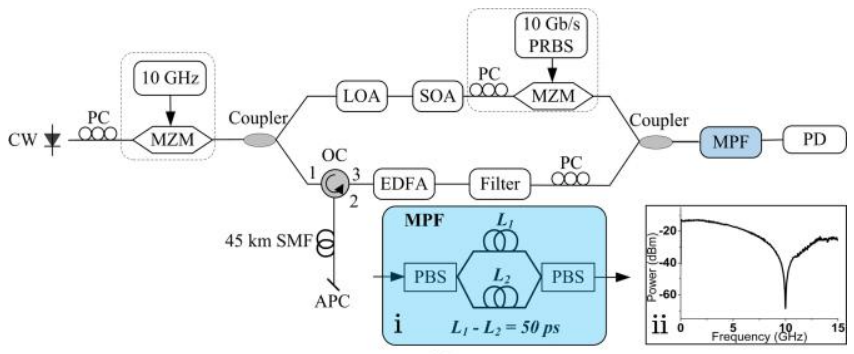

(a)

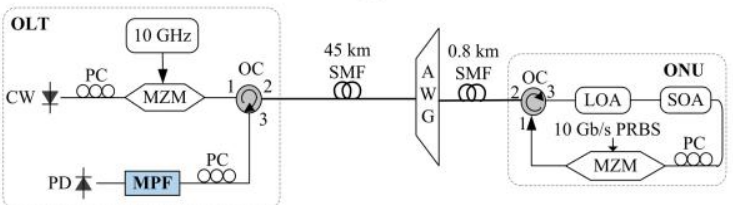

(b)

Fig. 4. Experimental setup (a) OSRNR and (b) BER performances. $\mathrm{CW}$ : continuous wave, $\mathrm{OC}$ : optical circulator, MZM: Mach-Zehnder modulator, SMF: single mode fiber, APC: angled physical contact, LOA: linear optical amplifier, SOA: semiconductor optical amplifier, PC: polarization controller, EDFA: erbium-doped fiber amplifier, MPF: microwave photonic filter, PD: photodiode. Insets of Fig. 4 (a): (i) systematical setup of MPF; (ii) frequency response of MPF.

To further improve the system performance, a MPF is placed before the receiver to suppress the residual microwave carrier and RB noise at around $10 \mathrm{GHz}$. The MPF structure is shown in the inset $\mathrm{i}$ of Fig. 4 (a), which consist of two polarization beam splitters (PBSs) and fiber delay lines. The path length difference (L1 - L2) is fixed at 50 ps which corresponding to a $10-\mathrm{GHz}$ notch filter in radio frequency domain. The MPF provides over $40 \mathrm{~dB}$ of suppression at $10 \mathrm{GHz}$ (inset ii of Fig. 4 (a)). MPF can be made to be polarization insensitive [21] by utilizing single-mode to multi-mode combiner [22] instead of PBS. Using the experimental setup as shown in Fig. 4 (a), we also study both the low frequency and high frequency beat noise in our system experimentally. For comparison, we first investigate the beating effect between a CW seeding light (upper branch) and the RB noise (lower branch) without applying any RB noise mitigation scheme (i.e. without 10 GHz modulation using the MZM and cascaded-SOA). Red curve in Fig. 5 (a) is the measured RF spectrum showing the level of beat noise at low frequency range from $0 \mathrm{GHz}$ to 0.2 GHz. With our RB noise mitigation scheme, the low 
frequency beat noise is shown by the green curve in Fig. 5 (a). CCSR of the carrier-suppressed signal is $21.7 \mathrm{~dB}$ with saturation output power at $10 \mathrm{dBm}$. Power of the carrier RB noise is $-12 \mathrm{dBm}$ that corresponds to $22 \mathrm{~dB}$ of OSRNR. Compare with the case when $\mathrm{CW}$ seeding light is used, low frequency interference from $0 \mathrm{GHz}$ to $0.2 \mathrm{GHz}$ is suppressed efficiently using the proposed central carrier suppression scheme. Our central carrier suppression scheme significantly reduces the power of the central carrier (CCSR of $21 \mathrm{~dB}$ ), resulting in a suppression of low frequency interference. On the other hand, due to the high-pass filtering effect of cascaded-SOA as shown in Fig. 3 (a), high frequency carriers of the multi-subcarrier signal are enhanced by the cascadedSOA. Thus, interference at high frequency region is increased after passing through the cascaded-SOA, especially at $10 \mathrm{GHz}$ where the modulation frequency is located. Figure 5 (b) shows the measured RF spectra at $10 \mathrm{GHz}$. Without applying RB noise mitigation scheme (i.e. $\mathrm{CW}$ seeding light is used without $10 \mathrm{GHz}$ modulation and cascaded-SOA), lowest noise level at $10 \mathrm{GHz}$ is achieved as shown by the red curve. When multi-subcarrier signal is used to realize central carrier suppression, high frequency interference occurs and is amplified, resulting in a strong $10 \mathrm{GHz} \mathrm{RF}$ tone with power above $-20 \mathrm{dBm}$ (blue curve). With the utilization of MPF with a transmission notch at $10 \mathrm{GHz}$, the residual $10 \mathrm{GHz}$ RF signal is suppressed by $30 \mathrm{~dB}$ (black curve).

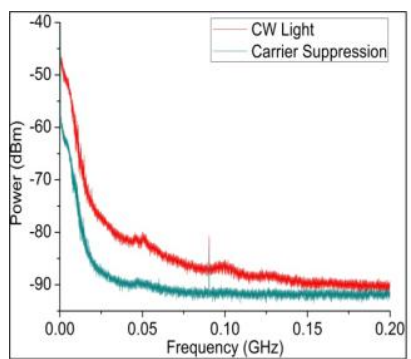

(a)

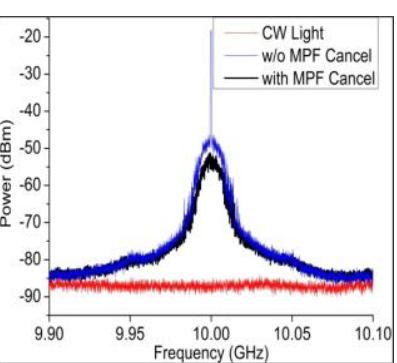

(b)
Fig. 5. (a) Low frequency electrical spectra, (b) High frequency regions.

Figure 6 (a) shows the measured power penalty of the upstream data at BER of 10-9 as a function of OSRNR by using the experimental setup depicted in Fig. 4 (a). When CW light is used as the downstream light without data modulation, power penalty at BER of $10-9$ is $1 \mathrm{~dB}$ as OSRNR reaches 22 $\mathrm{dB}$ (green inverted triangle curve). At this point, the receiver sensitivity is limited by the severe RB noise. With the proposed central carrier suppression scheme, error free transmission with no power penalty is achieved at OSRNR of $19.5 \mathrm{~dB}$ and CCSR of $8 \mathrm{~dB}$ (red circle curve). The RB noise tolerance is improved greatly by further increasing the CCSR value. When CCSR reaches $21 \mathrm{~dB}$, error free transmission with no power penalty is achieved when OSRNR is $15 \mathrm{~dB}$. Compared with the power penalty at $8 \mathrm{~dB}$ CCSR, RB noise tolerance is improved by $4.5 \mathrm{~dB}$ when CCSR is at $21 \mathrm{~dB}$. That is to say, low frequency interference is reduced with an improvement in upstream RB noise tolerance through the increase of central carrier suppression. Through using the experimental setup illustrated in Fig. 4 (b), the corresponding upstream BER performance is measured as shown in Fig. 6 (b). When CW light is used without modulation, error floor is observed in the BER measurement of the upstream signal and the corresponding eye diagram is shown in Fig. 6 (b) i. For an upstream signal with CCSR of $21 \mathrm{~dB}$, receiver sensitivity of $-24 \mathrm{dBm}$ is obtained (without using MPF). The corresponding eye diagram is shown in Fig. 6 (b) ii. Noise is found in the eye diagram due to the presence of residual downstream $10 \mathrm{GHz}$ signal. With the use of MPF for suppressing the residual 10 $\mathrm{GHz}$ signal and residual $\mathrm{RB}$ noise, receiver sensitivity is improved by $6 \mathrm{~dB}$. A widely open eye diagram is resulted as shown in Fig. 6 (b) iii.

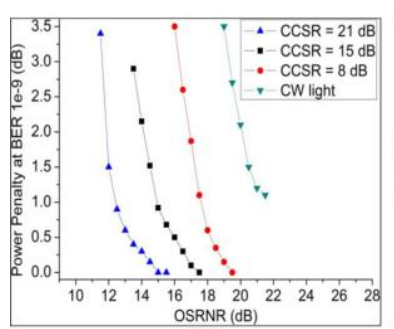

(a)

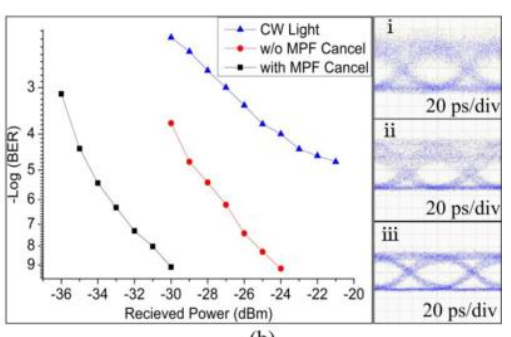

(b)
Fig. 6. Observed OSRNR graphs (a) BER curves (b) Related eye diagrams (right side $\mathrm{i} \sim$ iii).

\section{SIMULATION SETUP AND OPERATION}

The power budget analysis of the proposed system is also carried out, as shown in Table 1. In DS direction, the launching power output from the EDFA is set to be $8 \mathrm{dBm}$. The total loss is $18.5 \mathrm{~dB}$, including the power losses induced by an IL $(1 \mathrm{~dB}), 25-\mathrm{km}$ SMF transmission $(5 \mathrm{~dB})$, an optical circulator $(0.5 \mathrm{~dB})$, an AWG $(5 \mathrm{~dB})$ and an 80:20 OC $(7 \mathrm{~dB}$ for the $20 \%$ port). In US direction, since the output power of ROSA is decided by the power of input DS signal, we consider the case that the injected power is fixed at $\_12 \mathrm{dBm}$ and the RSOA has a saturated output power of $7.5 \mathrm{dBm}$. The total loss for US signal is $18 \mathrm{~dB}$ since the $80 \%$ port of $80: 20$ OC $(1 \mathrm{~dB})$ is connected with the RSOA and two $1_{-}$N AWGs as well as two circulators are traversed. The power margins for DS and US transmission are $19.3 \mathrm{~dB}$ and $20.2 \mathrm{~dB}$ respectively. Therefore, the proposed system could support larger transmission scope. We can also find that a $1: 64$ splitting ratio for each channel can be supported for hybrid WDM/TDM access. Note that when power splitter for TDM fashion is introduced, the DS signal power injected into the RSOA may be lower than the gain-saturation regime. This problem can be solved by using an additional SOA before the input of RSOA [23], [24].

Table 1. Power budget calculation for DS and US signals

\begin{tabular}{|c|c|c|}
\hline Element for power Budget & DS & UP \\
\hline $\begin{array}{l}\text { Lunching power after EDFA } \\
(\mathrm{dBm})\end{array}$ & 8 & - \\
\hline Injected power into RSOA (dBm) & - & -12 \\
\hline ROSA saturated output (dBm) & - & 7.9 \\
\hline Inter lever insertion loss $(\mathrm{dBm})$ & 1 & 1 \\
\hline 25 km SMF loss (dBm) & 5 & 5 \\
\hline $\begin{array}{l}\text { Optical circulator insertion loss } \\
(\mathrm{dBm})\end{array}$ & 0.5 & $0.5 \times 2$ \\
\hline $1 \times \mathrm{N}$ AWG insertion loss $(\mathrm{dBm})$ & $\begin{array}{l}7(20 \% \\
\text { port })\end{array}$ & $\begin{array}{l}1(80 \% \\
\text { port })\end{array}$ \\
\hline Total insertion loss $(\mathrm{dBm})$ & 18.6 & 18.1 \\
\hline Receiver sensitivity $(\mathrm{dBm})$ & -29.9 & -30.8 \\
\hline Power margin $(\mathrm{dBm})$ & 19.4 & 20.2 \\
\hline
\end{tabular}


Simulation setup and operation of proposed single fiber centralized light source bidirectional WDM-PON is shown in Fig. 7. In the central office (CO), 10-Gbps pseudorandom bit stream (PRBS) data of order 27-1 is used for 10 DQPSK channels downstream transmission, using ITU-T grid 100 $\mathrm{GHz}$ channel spacing, having launch power $5 \mathrm{dBm}$ generated by distributed feedback (DFB) laser sources ranging from 193.1 THz to $194.0 \mathrm{THz}$. The channels are multiplexed and transmitted over $25 \mathrm{~km}$ bidirectional standard single mode fiber (SSMF) as per Table-II

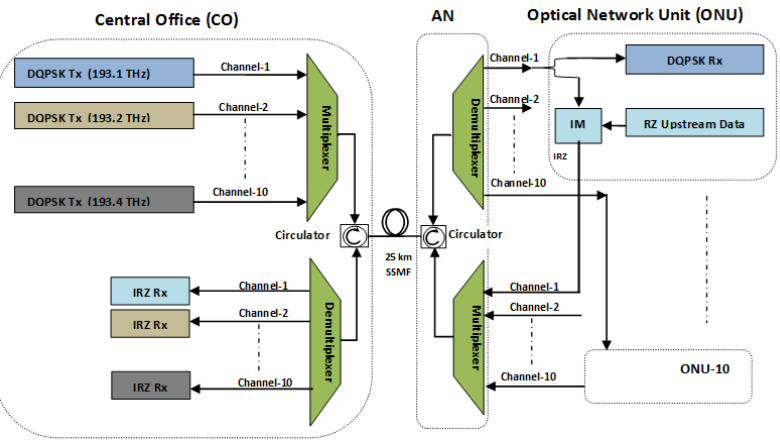

Fig. 7: Simulation \& operational setup of proposed 10 channels trans/receive WDM-PON

Table 2. Parameters used for simulation

\begin{tabular}{|l|c|}
\hline SSMF Parameters & Values \\
\hline Dispersion parameter & $16.75 \mathrm{ps} / \mathrm{nm} / \mathrm{km}$ \\
\hline Dispersion slope & $0.075 \mathrm{ps} / \mathrm{nm} 2 / \mathrm{km}$ \\
\hline Attenuation Coefficient & $0.2 \mathrm{~dB} / \mathrm{km}$ \\
\hline Effective core area & $80 \mathrm{um} 2$ \\
\hline $\begin{array}{l}\text { Non Linear index- } \\
\text { coefficient }\end{array}$ & $2.6 \times 10-20$ \\
\hline Rayleigh backscattering & $5 \times 10-5 / \mathrm{km}$ \\
\hline
\end{tabular}

Optical spectrum of 10 multiplexed downstream channels is shown in Fig. 8. After de-multiplexing, channels are fed in ONUs, where each channel is split in two parts by optical splitters. One part of the signal is fed to the DQPSK receiver for demodulation of downstream data and the other part is fed in upstream transmitter, in which downstream signal power is used for the re-modulation of IRZ upstream data via intensity modulator. In this way 10 upstream channels from ONUs are multiplexed and transmitted over $25 \mathrm{~km}$ SMF (without DCF as was in [25]) to- wards OLT in central office (CO), optical spectrum is shown in Fig. 9. 10 IRZ upstream channels are also multiplexed using ITU-T grid $100 \mathrm{GHz}$ channel spacing. At central office (CO), a simple PIN diode based IRZ receiver is used for upstream data reception for every wavelength.

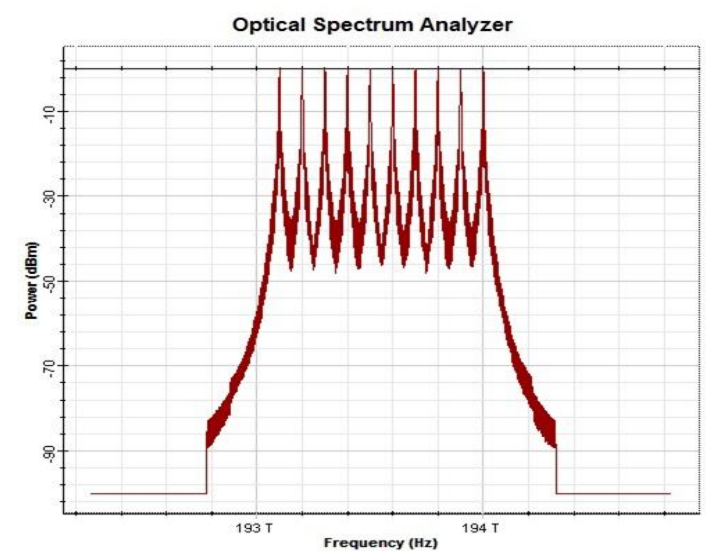

Fig. 8: Optical specta of 10 DQPSK channels

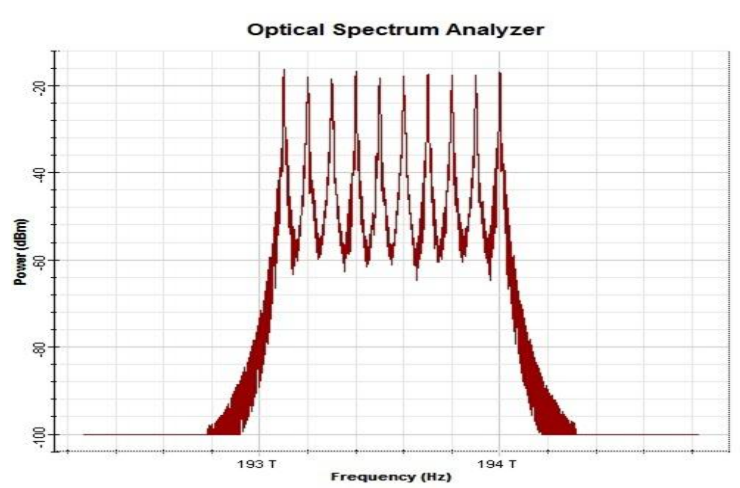

Fig. 9: Optical spectra of 10 IRZ channels.
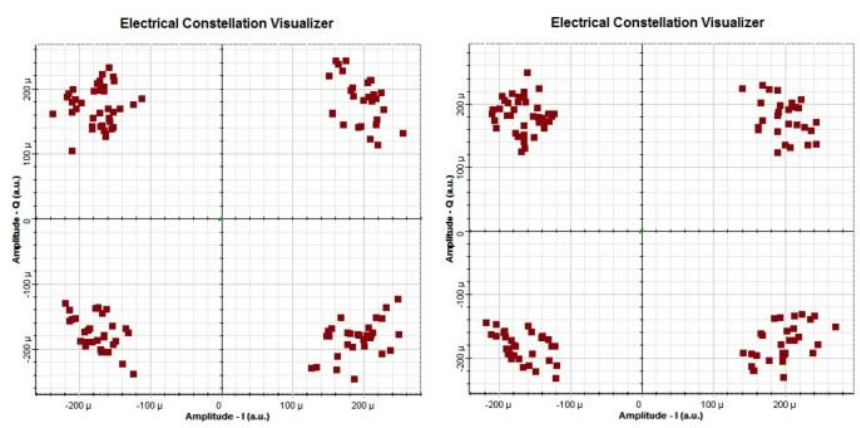

Fig. 10: Constellation chart for DQPSK channel 4 and 7
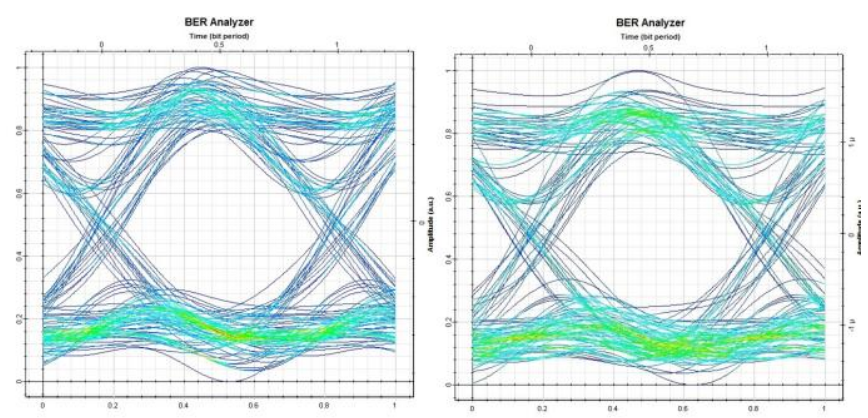

Fig. 11: Eye diagrams of DQPSK- I channel 4 and 7. 

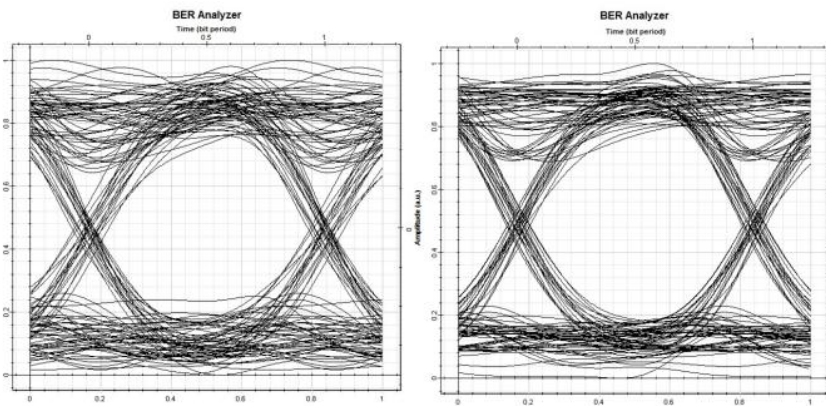

Fig. 12: Eye diagrams of DQPSK-Q channel 4 and 7.
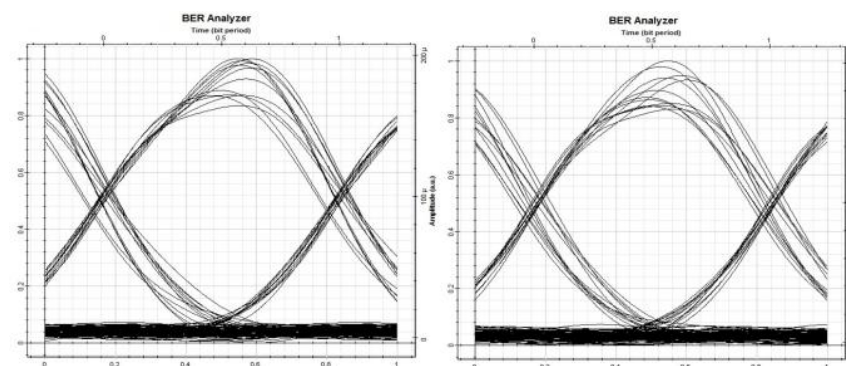

Fig. 13: Eye diagrams of IRZ channel 4 and 7.

\section{CONCLUSION}

In this paper, we have investigated carrier RB noise suppression performance by realizing central carrier suppression with cascaded-SOA. 10 DQPSK channels of 10 Gbit/s data rate are successfully transmitted in downstream without pulse carving, EDFA and DCF, whereas 10 IRZ channels of $2.5 \mathrm{Gbit} / \mathrm{s}$ data rate are successfully transmitted for upstream signal by re-modulation of downstream signals, no additional lasers have been used at optical network units (ONUs). Simulation results show that aggregated $100 \mathrm{Gbit} / \mathrm{s}$ downstream transmissions of 10 DQPSK channels and aggregated $25 \mathrm{Gbit} / \mathrm{s}$ upstream transmissions of $10 \mathrm{IRZ}$ channels, using $100 \mathrm{GHz}$ channel spacing, can be successfully achieved over a distance of $25 \mathrm{~km}$. Further it is observed that proposed single fiber based bidirectional WDM-PON has less than $1.5 \mathrm{dBm}$ transmission power penalties in all cases, which ensures high resilience against RB induced noise and also improved receiver sensitivity is obtained for all channels in downstream as well as upstream transmission.

\section{REFERENCES}

[1] G.-K. Chang, A. Chowdhury, Z. Jia, H.-C. Chien, M.-F. Huang, J. Yu, and G. Ellinas, "Key Technologies of WDM-PON for Future Converged Optical Broadband Access Networks [Invited]," J Opt. Commun. Netw. 1(4), C35-C50 (2009).

[2] Cisco Visual Networking Index: Forecast and Methodology", 2015- 2020. (Online) www.cisco.com

[3] Chun-Kit Chan, Lian-Kuan Chen et al. IWDM PON for Next-Generation Optical Broadband Access Networks", 5E2-1-1, ECOC (2006).

[4] E.Wong, ICurrent and next-generation broadband access technologies", presented at the OFC/NF- OEC, Los Angeles, CA, (2011).

[5] M. Fujiwara, J.-I. Kani, H. Suzuki, and K. Iwatsuki, "Impact of back reflection on upstream transmission in WDM single-fiber loopback access networks," J. Lightwave Technol. 24(2), 740-746 (2006).
[6] C. W. Chow, C. H. Yeh, L. Xu, and H. K. Tsang, "Rayleigh Backscattering Mitigation Using Wavelength Splitting for Heterogeneous Optical Wired and Wireless Access," IEEE Photon. Technol. Lett. 22(17), 12941296 (2010).

[7] Jae-Min Lee, Yong-Yuk Won et al, IBack-scattering noise reduction using RF tone in RSOA- based Optical Access Link", 2010 Wiley Periodicals, M/wave. Opt. Techno. Letter 53, pp. 331 \{ 334 (2011).

[8] Berrettini, Gianluca et al, IColorless WDM-PON Architecture for Rayleigh Backscattering and Path-Loss Degradation Mitigation", IEEE Photonics Technology Letters, Vol. 21, No. 7,(2009)

[9] J.Zhang, X.Yuan, et al. \A Novel Bidirectional RSOA based WDM-PON with downstream DPSK and upstream Re-Modulated OOK Data", ICTON (2009).

[10] L. Banchi, R. Corsini, M. Presi, et al, IEnhanced reection tolerance in WDM-PON by chirped RZ modulation", Electronics Letters, Vol. 46 No. 14, (2010).

[11] Shu-Chuan Lin, San-Liang Lee, et al, ICross-Seeding Schemes for WDM-Based Next- eneration Optical Access Networks", Journal Of Lightwave Technology, VOL. 29, NO. 24, (2011).

[12] C. H. Yeh and Chow, ISignal Remodulation Ring WDM Passive Optical Network with Rayleigh Backscattering Interferometric Noise Mitigation", IEEE Comm. Letter, VOL. 15, NO. 10, (2011)

[13] Jing Xu, Ming Li, et al, \Rayleigh Noise Reduction in $10-\mathrm{Gb} / \mathrm{s}$ Carrier-Distributed WDM-PONs Using In-Band Optical Filtering", Journal Of Lightwave Technology, VOL. 29, NO. 24 (2011).

[14] Andrea Chiuchiarelli, Presi, et al \Enhancing resilience to Rayleigh crosstalk by means of line coding and electrical _ltering", IEEE Photonics Technology Letter, 22, (2), pp. $85\{87,(2010)$.

[15] Y. Khan, YU Chong et al, IRayleigh-Backscattering Minimization in Single Fiber Colorless WDM- PON using Intensity Re-modulation Technique", Optoelectronics Letters Vol. 8 No. 5, Sept (2012).

[16] A. Nakanishi, N. Sasada, Y. Sakuma, et al., "Uncooled $\left(0\right.$ to $\left.85^{\circ} \mathrm{C}\right)$ and Full C-band Operation of a $10.7 \mathrm{Gbit} / \mathrm{s}$ InP Mach-Zehnder Modulator Monolithically Integrated with SOA," in Optical Fiber Communication Conference (OFC), 2013, paper OW1G.3.

[17] Infinera white paper, "Photonic Integrated Circuits," http://www.infinera.com

[18] S. Porto, C. Antony, P. Ossieur, and P. D. Townsend, "An upstream reach-extender for $10 \mathrm{~Gb} / \mathrm{s}$ PON applications based on an optimized semiconductor amplifier cascade," Opt. Express 20(1), 186-191 (2012).

[19] K. Sato and H. Toba, "Reduction of mode partition noise by using semiconductor optical amplifiers," IEEE J. Sel. Top. Quantum Electron. 7(2), 328-333 (2001).

[20] J. Chang, M. P. Fok, J. Meister, and P. R. Prucnal, "A single source microwave photonic filter using a novel single-mode fiber to multimode fiber coupling technique," Opt. Express 21(5), 5585-5593 (2013). 
[21] M. P. Fok, Y. Deng, K. Kravtsov, and P. R. Prucnal, "Signal beating elimination using single-mode fiber to multimode fiber coupling," Opt. Lett. 36(23), 4578-4580 (2011)

[22] I. N. Cano, M. Omella, J. Prat, and P. Poggiolini, BColorless $10 \mathrm{~Gb} / \mathrm{s}$ extended reach WDM PON with low BW RSOA using MLSE, [presented at the Opt. Fiber Commun. Conf. (OFC), San Diego, CA, USA, 2010, Paper OWG2.

[23] Y.-H. Lin, C.-J. Lin, G.-C. Lin, and G.-R. Lin, BSaturated signal-to-noise ratio of up-stream WRCFPLD transmitter injection-locked by down-stream dataerased ASE carrier,[ Opt. Exp., vol. 19, no. 5, pp. 40674075, Feb. 2011.

[24] ZHANG Wei-feng , Xin Xiang-jun et al. ICentralized light-wave WDM-PON employing DQPSK downstream and OOK remodulated upstream signals", Elsevier, Journal of China Universities Post and Telecom. 10058885, 17(4): pp. 125 \{ 128 August (2010).
[25] Zhao Zhou, Shilin Xiao, Tao Qi, Pingqing Li, Meihua Bi, Weisheng $\mathrm{Hu}$ "25-GHz-Spaced DWDM-PON With Mitigated Rayleigh Backscattering and Back-Reflection Effects" IEEE Photonics Journal Volume 5, Number 4, August 2013

[26] Hanlin Feng, Jia Ge, Shilin Xiao, and Mable P. Fok, ISuppression of Rayleigh backscattering noise using cascaded-SOA and microwave photonic filter for 10 Gb/s loop-back WDM-PON" in OPTICS EXPRESS , MAY 2014

[27] Y. Khan, YU Chong et al, IRayleigh-Backscattering Minimization in Single Fiber Colorless WDM-PON using Intensity Re-modulation Technique", Optoelectronics Letters Vol. 8 No. 5, Sept (2012).

[28] C. W. Chow, C. H. Yeh, "Using Downstream DPSK and Upstream Wavelength-Shifted ASK for Rayleigh Backscattering Mitigation in TDM- PON to WDM-PON Migration Scheme" IEEE Photonics Journal Volume 5, Number 2, April 2013. 